\title{
On the contact equivalence problem of second order ODEs which are quadratic with respect to the second order derivative
}

\author{
Vadim V. Shurygin, jr. \\ Kazan (Volga Region) Federal University, Russia \\ e-mail: vshjr@yandex.ru, vadimjr@kpfu.ru
}

\begin{abstract}
In the present paper we establish the necessary and sufficient conditions for two ordinary differential equations of the form $y^{\prime \prime 2}+A\left(x, y, y^{\prime}\right) y^{\prime \prime}+B\left(x, y, y^{\prime}\right)=0$ to be equivalent under the action of the pseudogroup of contact transformations. These conditions are formulated in terms of integrals of some one-dimensional distributions.
\end{abstract}

Keywords: contact transformations, point transformations, differential invariants, ODEs.

\section{Introduction}

In the present paper we solve the problem of equivalence of 2 nd order ODEs of the form

$$
y^{\prime \prime 2}+A\left(x, y, y^{\prime}\right) y^{\prime \prime}+B\left(x, y, y^{\prime}\right)=0
$$

under the action of the pseudogroup of contact transformations of the space $\mathbb{R}^{3}(x, y, p)$, where $p=y^{\prime}$. We use the results of A. Tresse and B. Kruglikov who described the algebra of differential invariants of 2 nd order ODEs of the form

$$
y^{\prime \prime}=f\left(x, y, y^{\prime}\right)
$$

under the action of the pseudogroup of point transformations of the space $\mathbb{R}^{2}(x, y)$.

S. Lie showed that any two second order ODEs of the form (2) are equivalent under the action of contact transformations pseudogroup of the space $\mathbb{R}^{3}(x, y, p)$. The problem of point equivalence of such ODEs was the point of investigation of many papers. Lie proved that if the ODE (2) is point equivalent to the linear equation $y^{\prime \prime}=0$, it is necessary cubic with respect to the first derivative, i.e., is of the form

$$
y^{\prime \prime}=a_{3}(x, y) y^{\prime 3}+a_{2}(x, y) y^{\prime 2}+a_{1}(x, y) y^{\prime}+a_{0}(x, y) .
$$

The class of such equations is closed under the action of point transformations pseudogroup. Moreover, he formulated the sufficient conditions of the ODE to be linearizable (see [5]). R. Liouville [6] found precise conditions for linearization. A. Tresse [9] found the complete set of relative differential invariants of ODEs (2). B. Kruglikov [4] formulated the results of Tresse in a modern language. He described the algebra of absolute differential invariants and solved the problem of equivalence of such ODEs under the action of point transformations pseudogroup. The class of ODEs, cubic with respect to the first derivatives was considered by Tresse [8], who also found the relative invariants of these ODEs. V. Yumaguzhin $[10,11]$ studied the the problem of point equivalence of such ODEs and the obstructions to linearization. We also note the book of N. Ibragimov [2], the paper of N. Ibragimov and F. Magri [3] and the paper of O. Morozov [7]. 


\section{Preliminaries}

Let $\pi$ be a vector bundle and $G$ be the pseudogroup of diffeomorphisms acting on $\pi$. The action of $G$ naturally prolongs to the action on the space $J^{k} \pi$ of $k$-jets of the sections of $\pi$. The function $I \in C^{\infty}\left(J^{k} \pi\right)$ is called an (absolute) scalar differential invariant of order $k$ if it is constant along the orbits of prolonged action of $G$ on $J^{k} \pi$. The set of all absolute differential invariants is an algebra $\mathcal{A}$ with respect to the standard arithmetic operations.

The function $F \in C^{\infty}\left(J^{k} \pi\right)$ is called a relative scalar differential invariant of order $k$ if for every $g \in G$ one has $g^{*} F=\mu(g) \cdot F$, for a smooth function $\mu: G \rightarrow C^{\infty}\left(J^{k} \pi\right)$ satisfying the conditions $\mu(g \cdot h)=h^{*} \mu(g) \cdot \mu(h), \mu(e)=1$. In other words, the equation $F=0$ is invariant under the action of $G$. The function $\mu$ is called a weight. Let $\mathcal{M}=\{\mu\}$ be the space of all weights. Denote by $\mathcal{R}^{\mu}$ the space of relative differential invariants of weight $\mu$. Then $\mathcal{R}=\bigcup_{\mu \in \mathcal{M}} \mathcal{R}^{\mu}$ is a $\mathcal{M}$-graded module over the algebra of absolute differential invariants. An invariant differential operator is a combination of total derivatives $\mathcal{D}_{x^{i}}$ in base coordinates $\left(x^{1}, \ldots, x^{n}\right)$ of $\pi$

$$
\Delta=\sum_{i=1}^{n} A_{i} \mathcal{D}_{x^{i}}+A, \quad A_{i}, A \in C^{\infty}\left(J^{k} \pi\right),
$$

which commutes with the action of $G$.

Let us formulate the classification of Tresse and Kruglikov which we will need in what follows.

Any ODE $y^{\prime \prime}=f\left(x, y, y^{\prime}\right)$ may be represented as a section of the bundle $J^{0} \mathbb{R}^{3}(x, y, p)=$ $\mathbb{R}^{4}(x, y, p, q)$, where $p=y^{\prime}, q=y^{\prime \prime}$ denote the corresponding coordinates in jet space. Let $\mathcal{D}_{x}$, $\mathcal{D}_{y}, \mathcal{D}_{p}$ be the total derivatives in $x, y$ and $p$ respectively. We also denote $\hat{\mathcal{D}}_{x}=\mathcal{D}_{x}+p \mathcal{D}_{y}$. Let $q_{l m}^{k}=\hat{\mathcal{D}}_{x}^{l} \mathcal{D}_{y}^{m} \mathcal{D}_{p}^{k}(q)$.

The weights form a two-dimensional lattice and the space of relative invariants is a direct sum $\mathcal{R}=\bigoplus_{r, s \in \mathbb{Z}} \mathcal{R}^{r, s}$ (see [4]). First relative invariants appear in order 4 , they are

$$
\begin{aligned}
I=q_{00}^{4}, \quad H=q_{20}^{2}-4 q_{11}^{1}+6 q_{02}^{0}+q( & \left.2 q_{10}^{3}-3 q_{01}^{2}\right)- \\
& -q_{00}^{1}\left(q_{10}^{2}-4 q_{01}^{1}\right)+q_{00}^{3} q_{10}^{0}-3 q_{00}^{2} q_{01}^{0}+q \cdot q \cdot q_{00}^{4} .
\end{aligned}
$$

Invariant differential operators are

$$
\begin{aligned}
\Delta_{p}= & \mathcal{D}_{p}+(r-s) \frac{q_{00}^{5}}{5 q_{00}^{4}}: \mathcal{R}^{r, s} \rightarrow \mathcal{R}^{r-1, s+1} \\
\Delta_{x}= & \mathcal{D}_{x}+p \mathcal{D}_{y}+q \mathcal{D}_{p}+r\left(3 q_{00}^{1}+2 \frac{q_{00}^{5} q+q_{10}^{4}}{q_{00}^{4}}\right)+ \\
& +s\left(2 q_{00}^{1}+\frac{q_{00}^{5} q+q_{10}^{4}}{q_{00}^{4}}\right): \mathcal{R}^{r, s} \rightarrow \mathcal{R}^{r+1, s} \\
\Delta_{y}= & \frac{q_{00}^{5}}{5 q_{00}^{4}} \mathcal{D}_{x}+\left(1+p \frac{q_{00}^{5}}{5 q_{00}^{4}}\right) \mathcal{D}_{y}+\left(2 q_{00}^{1}+\frac{5 q_{10}^{4}+6 q_{00}^{5} q}{q_{00}^{4}}\right) \mathcal{D}_{p}+ \\
& +r\left(\frac{3 q_{00}^{2}}{8}+\frac{q_{01}^{4}}{4 q_{00}^{4}}+\frac{19 q_{00}^{1} q_{00}^{5}}{10 q_{00}^{4}}+\frac{21\left(q_{00}^{5} q+q_{01}^{4}\right) q_{00}^{5}}{20 q_{00}^{4} q_{00}^{4}}\right)+ \\
& +s\left(\frac{q_{00}^{2}}{4}+\frac{q_{01}^{4}}{2 q_{00}^{4}}+\frac{3 q_{00}^{1} q_{00}^{5}}{5 q_{00}^{4}}+\frac{3\left(q_{00}^{5} q+q_{01}^{4}\right) q_{00}^{5}}{10 q_{00}^{4} q_{00}^{4}}\right): \mathcal{R}^{r, s} \rightarrow \mathcal{R}^{r, s+1} .
\end{aligned}
$$

We say that the equation $y^{\prime \prime}=f\left(x, y, y^{\prime}\right)$ is generic, if $I H \neq 0$ for it. 
Theorem 1. [4] The space of relative differential invariants of a generic equation $y^{\prime \prime}=$ $f\left(x, y, y^{\prime}\right)$ is generated by an invariant $H$ and the invariant differential operators $\Delta_{x}, \Delta_{y}, \Delta_{p}$.

We also formulate the equivalence theorem for the generic ODEs.

The functions

$$
H_{10}=\Delta_{x} H, \quad H_{01}=\Delta_{y} H, \quad K=\Delta_{p} H
$$

form the basis of invariants of order 5 . There are 11 basic invariants in order 6 , we need 8 out of them, namely

$$
\begin{aligned}
& H_{20}=\Delta_{x}^{2} H, \quad H_{11}=\Delta_{x} \Delta_{y} H, \quad H_{02}=\Delta_{y}^{2} H, \quad K_{10}=\Delta_{x} K, \quad K_{01}=\Delta_{y} K, \\
& \Omega^{6}=q_{00}^{6}-\frac{6}{5} \cdot \frac{q_{00}^{5} \cdot q_{00}^{5}}{q_{00}^{4}}, \quad \Omega_{10}^{5}=\frac{5 I}{24 H}\left(\left[\Delta_{p}, \Delta_{x}\right] H-\Delta_{y} H\right), \quad \Omega_{20}^{4}=\Delta_{p}^{2} H-\frac{\Omega^{6}}{5 I} H .
\end{aligned}
$$

We also denote

$$
J_{1}=I^{-1 / 8} H^{3 / 8} \in \mathcal{R}^{1,0}, \quad J_{2}=I^{1 / 4} H^{1 / 4} \in \mathcal{R}^{0,1} .
$$

Using $J_{1}$ and $J_{2}$ construct the following invariants of pure order 5 and 6 :

$$
\begin{array}{lll}
\bar{H}_{10}=H_{10} /\left(J_{1}^{3} J_{2}\right), & \bar{H}_{01}=H_{01} /\left(J_{1}^{2} J_{2}^{2}\right), & \bar{K}=K /\left(J_{1} J_{2}^{2}\right), \\
\bar{H}_{20}=H_{20} /\left(J_{1}^{4} J_{2}\right), & \bar{H}_{11}=H_{11} /\left(J_{1}^{3} J_{2}^{2}\right), & \bar{H}_{02}=H_{02} /\left(J_{1}^{2} J_{2}^{3}\right), \\
\bar{K}_{10}=K_{10} /\left(J_{1}^{2} J_{2}^{2}\right), & \bar{K}_{01}=K_{01} /\left(J_{1} J_{2}^{3}\right), & \\
\bar{\Omega}^{6}=\Omega^{6} /\left(J_{1}^{-4} J_{2}^{5}\right), & \bar{\Omega}_{10}^{5}=\Omega_{10}^{5} /\left(J_{1}^{-2} J_{2}^{4}\right), & \bar{\Omega}_{20}^{4}=\Omega_{20}^{4} /\left(J_{2}^{3}\right) .
\end{array}
$$

Any second order ODE $\mathcal{E}$ may be considered as a section $\mathfrak{s}_{\mathcal{E}}$ of a bundle $J^{0} \mathbb{R}^{3}(x, y, p)$. Hence, any differential invariant $I$ of order $k$ may be restricted to the equation $\mathcal{E}$ via pull-back of the $k$ th prolongation of the section $\mathfrak{s}_{\mathcal{E}}$ :

$$
I^{\mathcal{E}}:=\left(\mathfrak{s}_{\mathcal{E}}^{(k)}\right)^{*}(I)
$$

Let $\mathcal{E}$ be a generic second order ODE such that the functions $\bar{H}_{10}^{\mathcal{E}}, \bar{H}_{01}^{\mathcal{E}}, \bar{K}^{\mathcal{E}}$ are local coordinates on $\mathbb{R}^{3}(x, y, p)$. The other differential invariants can be expressed as functions of these three:

$$
\bar{H}_{i j}^{\mathcal{E}}=\Phi_{i j}^{\mathcal{E}}\left(\bar{H}_{10}^{\mathcal{E}}, \bar{H}_{01}^{\mathcal{E}}, \bar{K}^{\mathcal{E}}\right), \quad \bar{K}_{i j}^{\mathcal{E}}=\Psi_{i j}^{\mathcal{E}}\left(\bar{H}_{10}^{\mathcal{E}}, \bar{H}_{01}^{\mathcal{E}}, \bar{K}^{\mathcal{E}}\right), \quad \bar{\Omega}_{i j}^{k \mathcal{E}}=\Upsilon_{i j}^{k \mathcal{E}}\left(\bar{H}_{10}^{\mathcal{E}}, \bar{H}_{01}^{\mathcal{E}}, \bar{K}^{\mathcal{E}}\right)
$$

Theorem 2. [4] Two generic second order ODEs $\mathcal{E}_{1}$ and $\mathcal{E}_{2}$ are equivalent under the action of the pseudogroup of point transformations if and only if the functions

$$
\Phi_{20}^{\mathcal{E}}, \Phi_{11}^{\mathcal{E}}, \Phi_{02}^{\mathcal{E}}, \Psi_{10}^{\mathcal{E}}, \Psi_{01}^{\mathcal{E}}, \Upsilon^{6 \mathcal{E}}, \Upsilon_{10}^{5 \mathcal{E}}, \Upsilon_{20}^{4 \mathcal{E}}
$$

coincide.

The criteria for equivalence of non-generic ODEs can be found in [4].

\section{Various formulations of the problem}

Let $M$ be a 3 -dimensional contact manifold with the contact distribution $\mathcal{C}$. Let $(x, y, p)$ denote the canonical coordinates in $M$. Then $\mathcal{C}$ is determined by a contact 1 -form $\omega=d y-p d x$. 
We will say the the ODE (1) is of hyperbolic type if $D=A^{2}-4 B>0$, and that it is of of elliptic type if $D<0$. Since we are interested in the real case, we restrict ourselves to the ODEs of hyperbolic type. Let

$$
y^{\prime \prime}=\lambda_{1}\left(x, y, y^{\prime}\right) \quad \text { and } \quad y^{\prime \prime}=\lambda_{2}\left(x, y, y^{\prime}\right)
$$

be the roots of this ODE. Note that $D$ is a relative contact invariant on the set of ODEs (1).

Geometrically, each of the ODEs $y^{\prime \prime}=\lambda_{i}\left(x, y, y^{\prime}\right)$ determines a 1-dimensional distribution $\mathcal{F}_{i}$ in $\mathbb{R}^{3}(x, y, p)=J^{0} \mathbb{R}^{2}(x, y)$. This distribution lies in $\mathcal{C}$ and is spanned by a vector field

$$
X_{i}=\frac{\partial}{\partial x}+p \frac{\partial}{\partial y}+\lambda_{i}(x, y, p) \frac{\partial}{\partial p} .
$$

The Lie bracket $\left[X_{1}, X_{2}\right]$ does not belong to $\mathcal{C}$.

Definition. We will say that two 1 -dimensional distributions $\mathcal{F}_{1}$ and $\mathcal{F}_{2}$ in a 3 -dimensional manifold $M$ are in general position if

1) vector fields $X, Y$ which define these distributions, are linear independent at any point;

2) their Lie bracket $[X, Y]$ is linear independent with $X, Y$.

Note that the 2-dimensional distribution $\mathcal{F}_{1} \oplus \mathcal{F}_{2}$ determines the contact structure on $M$.

Theorem 3. The ODE (1) of hyperbolic type is equivalent to a pair of 1-dimensional distributions in general position in $\mathbb{R}^{3}(x, y, p)$.

Proof. The necessity is obvious.

Let $\mathcal{F}_{1}$ and $\mathcal{F}_{2}$ be two 1 -dimensional distributions in general position in $\mathbb{R}^{3}(x, y, p)$ and let $X, Y$ be vector fields determining these distributions. Denote $\mathcal{F}=\mathcal{F}_{1} \oplus \mathcal{F}_{2}$. Let us prove that the condition 2) of the definition does not depend on a choice of the basis in $\mathcal{F}$. For any functions $f$ and $g$ we have

$$
[f X, g Y]=f g \cdot[X, Y]+f \cdot X g \cdot Y-g \cdot Y f \cdot X=f g \cdot[X, Y] \bmod \mathcal{F} .
$$

It follows that for another basis $X^{\prime}=\alpha X+\beta Y, Y^{\prime}=\gamma X+\delta Y$ one has

$$
\left[X^{\prime}, Y^{\prime}\right]=(\alpha \delta-\beta \gamma) \cdot[X, Y] \neq 0 \bmod \mathcal{F},
$$

since $[X, Y] \neq 0 \bmod \mathcal{F}, \alpha \delta-\beta \gamma \neq 0$.

Let $a, b$ be independent integrals of $X$ and $f, g$ be independent integrals of $Y$. Since $X$ and $Y$ are linear independent, any three of these functions are independent and the fourth one can be expressed in terms of these three. Let, for example, $g=h(a, b, f)$. Choose the functions $a, b$, $f$ as local coordinates. In these coordinates the fields $X$ and $Y$ (up to the factors) have the form

$$
X=\frac{\partial}{\partial f}, \quad Y=h_{b} \frac{\partial}{\partial a}-h_{a} \frac{\partial}{\partial b}
$$

Note that $h_{a} \neq 0$, since $b, f, g$ are independent. Similarly, $h_{b} \neq 0$. Denote $c=-h_{a} / h_{b}$. Since

$$
[X, Y]=h_{b f} \frac{\partial}{\partial a}-h_{a f} \frac{\partial}{\partial b}
$$

does not belong to $\mathcal{F}$, one has

$$
h_{a} h_{b f}-h_{b} h_{a f} \neq 0
$$


This is equivalent to the inequality

$$
\frac{\partial}{\partial f}\left(\frac{h_{a}}{h_{b}}\right) \neq 0
$$

which means that one can choose $a, b, c$ as coordinates on $\mathbb{R}^{3}$. In these coordinates $X, Y$ will have the form (up to the factors)

$$
X=\frac{\partial}{\partial c}, \quad Y=\frac{\partial}{\partial a}+c \frac{\partial}{\partial b}-\frac{\operatorname{Flex}(h)}{h_{b}^{3}} \frac{\partial}{\partial c},
$$

where we denoted

$$
\operatorname{Flex}(h)=h_{a a} h_{b}^{2}-2 h_{a} h_{b} h_{a b}+h_{b b} h_{a}^{2} .
$$

The distribution $\mathcal{F}$ is determined by the 1 -form $\omega=d b-c d a$ and defines the contact structure on $\mathbb{R}^{3}$.

Note that if a vector field $Z$ lies in $\mathcal{F}$ and is linear independent with $X$, then up to a factor it is of the form (6). Consider the contact transformation which satisfies the following conditions: 1) it preserves some point in $\mathbb{R}^{3}, 2$ ) its differential is sufficiently close to identity and 3) the direction of the field $X$ is not an eigendirection. Such a transformation maps $X$ and $Y$ to the fields which are not proportional to $X$. These vector fields up to the factors will have the form (6). Thus, they determine two equations (5).

Let us also show that the ODE (1) can be treated as two pairs of functions on $M$, satisfying some additional conditions.

The equation $y^{\prime \prime}=\lambda_{1}\left(x, y, y^{\prime}\right)$ locally has a pair of integrals $(a, b)$ (which are also integrals of $X_{1}$ ) such that $d a \wedge d b \neq 0$. Since $X_{1}$ lies in the kernel of the forms $\omega$, $d a$ and $d b$, one has $\omega=\alpha d a+\beta d b$ for some functions $\alpha, \beta$. Thus, ODE (1) of hyperbolic type, determines two pairs of functions $(a, b)$ and $(f, g)$ such that any three of the are independent. It follows from the equality $d a\left(X_{1}\right)=X_{1} a$ that 1 -forms $\left(X_{1} g\right) d a-\left(X_{1} f\right) d b \quad\left(X_{2} b\right) d f-\left(X_{2} a\right) d g$ are proportional to $\omega$.

Definition. Let $(a, b)$ and $(f, g)$ be two pairs of functions on $M$. We will say that they are in general position, if:

1) any three of them are independent;

2) the 1-dimensional distributions determined by $X$ and $Y$ are in general position.

Proposition 1. Let two pairs of functions $(a, b)$ and $(f, g)$ on $M$ be in general position. Let $a^{\prime}=a^{\prime}(a, b), b^{\prime}=b^{\prime}(a, b)$ be independent functions, and $f^{\prime}=f^{\prime}(f, g), g^{\prime}=g^{\prime}(f, g)$ be independent functions. Then the pairs $\left(a^{\prime}, b^{\prime}\right)$ and $\left(f^{\prime}, g^{\prime}\right)$ also are in general position.

Proof. Follows from the fact that both pairs of functions $(a, b)$ and $\left(a^{\prime}, b^{\prime}\right)$ determine the same distributions.

Theorem 4. The ODE (1) of hyperbolic type is equivalent to two pairs of functions $(a, b)$, $(f, g)$ on $M$ in general position. 


\section{The main result}

Now we return to the ODEs $y^{\prime \prime}=\lambda_{i}\left(x, y, y^{\prime}\right)$ and vector fields $X_{i}, i=1,2$. Choose two pairs of independent integrals $(a, b)$ and $(f, g)$ of $X_{1}$ and $X_{2}$ respectively. Let $g=h(a, b, f)$. Let

$$
a, b, c=-\frac{h_{a}}{h_{b}}
$$

be new coordinates in $\mathbb{R}^{3}$ and let $G_{2}(a, b, c)=-$ Flex $(h) / h_{b}^{3}$. Then $X_{1}$ and $X_{2}$ will have the form

$$
X_{1}=\frac{\partial}{\partial c} \text { and } X_{2}=\frac{\partial}{\partial a}+c \frac{\partial}{\partial b}+G_{2}(a, b, c) \frac{\partial}{\partial c},
$$

respectively. We will say that $(a, b, c)$ are canonical coordinates and that the ODE

$$
b^{\prime \prime}=G_{2}\left(a, b, b^{\prime}\right), \quad b=b(a),
$$

determined by $X_{2}$ is associated with the ODE (1).

Let $M_{3}$ and $\widetilde{M}_{3}$ be two 3-dimensional contact manifolds. Consider the contact transformation $\varphi: M_{3} \rightarrow \widetilde{M}_{3}$ which maps the ODE (1) into another equation of the same form. Let it map the vector field $X_{1}$ to the vector field $\widetilde{X}_{1}=\varphi^{*} X_{1}$. We prove that $\varphi$ acts of associated equations as a point transformation.

Let $M_{2}=M_{3} / X_{1}$ be the factor of $M_{3}$ by the trajectories of $X_{1}$ (locally it can be done). Let $\pi: M_{3} \rightarrow M_{2}$ be the canonical projection. Similarly, let $\widetilde{\pi}: \widetilde{M}_{3} \rightarrow \widetilde{M}_{2}=\widetilde{M}_{3} / \widetilde{X}_{1}$. The modelling bundle for $\pi$ is the bundle $\pi_{0}: J^{1} N \rightarrow J^{0} N$ where $N$ is some 1-dimensional manifold.

Let $\varphi_{0}: J^{1} N \rightarrow J^{1} \widetilde{N}$ be the restriction on $\varphi$ to $J^{1} N$. The diffeomorphism $\varphi$ maps the integrals $a$ and $b$ of the distribution determined by $X_{1}$ to some integrals $\widetilde{a}$ and $\widetilde{b}$ of the distribution determined by $\widetilde{X}_{1}$, i.e., to functions of $a$ and $b$. Thus, it induces the diffeomorphism $\psi_{0}: J^{0} N \rightarrow$ $J^{0} \widetilde{N}$, which closes the diagram



Since one can choose the functions $a$ and $b$ as coordinates on $J^{0} N$, one can treat $\psi_{0}$ as a point transformation. Conversely, any diffeomorphism $\psi_{0}: J^{0} N \rightarrow J^{0} \widetilde{N}$ lifts to the contact diffeomorphism $\varphi_{0}: J^{1} N \rightarrow J^{1} \tilde{N}$. Hence, the canonical coordinate system is defined up to a change of $(a, b)$ to the pair of independent functions $(\widetilde{a}(a, b), \widetilde{b}(a, b))$.

Proposition 2. The point equivalence class of the associated equation (8) does not depend on the choice of the integrals $(a, b),(f, g)$.

Proof. The choice of another integrals $\widetilde{a}, \widetilde{b}$ of $X_{1}$ instead of $a, b$ induces the change of canonical coordinates, thus does not change the equivalence class of the ODE (8).

Let $F(a, b, f, g)=0$ denote some functional dependency between these integrals. Any other dependency (in particular, $g-h(a, b, f)=0$ ) has the form

$$
\widetilde{F}(a, b, f, g)=\Phi(F(a, b, f, g))=0 .
$$


Clearly, the ratio $\widetilde{F}_{a} / \widetilde{F}_{b}$ does not depend on $\Phi$, in particular,

$$
\frac{F_{a}}{F_{b}}=\frac{h_{a}}{h_{b}}
$$

The choice of another integrals $\tilde{f}=\phi(f, g) \quad \tilde{g}=\psi(f, g)$ also does not change the ratio $F_{a} / F_{b}$, hence, does not change the canonical coordinate system.

In the construction above we can change the order of $X_{1}$ and $X_{2}$. Thus we obtain another ODE $b^{\prime \prime}=G_{1}\left(a, b, b^{\prime}\right)$, associated with (1). We say that these ODEs are dual to each other (see [1]).

From above it follows the

Theorem 5. Let $\mathcal{E}$ and $\widetilde{\mathcal{E}}$ be two ODEs (1) of hyperbolic type and let $\left(\left[\mathcal{E}_{1}^{a}\right],\left[\mathcal{E}_{2}^{a}\right]\right)$ and $\left(\left[\widetilde{\mathcal{E}}_{1}^{a}\right],\left[\widetilde{\mathcal{E}}_{2}^{a}\right]\right)$ be the pairs of point equivalence classes of their associated ODEs. The ODEs $\mathcal{E}$ and $\widetilde{\mathcal{E}}$ are contact equivalent if and only if one of the classes $\left(\left[\mathcal{E}_{1}^{a}\right],\left[\mathcal{E}_{2}^{a}\right]\right)$ coincides with one of the classes $\left(\left[\widetilde{\mathcal{E}}_{1}^{a}\right],\left[\widetilde{\mathcal{E}}_{2}^{a}\right]\right)$.

Let us present some examples.

Example 1. Consider the equation

$$
y^{\prime \prime 2}-y^{\prime \prime}=0 .
$$

Here $\lambda_{1}=1, \lambda_{2}=0$. Let

$$
a=p-x, \quad b=\frac{1}{2} x^{2}-p x+y, \quad f=p, \quad g=y-p x .
$$

Then $g=b-\frac{1}{2}(a-f)^{2}$, which yields $G_{2}=1$. On the other hand, $b=g+\frac{1}{2}(a-f)^{2}$, which yields $G_{1}=-1$. Thus, the pair of ODEs associated with (9) are

$$
b^{\prime \prime}=1 \quad \text { and } \quad b^{\prime \prime}=-1 .
$$

Clearly, they are point equivalent.

Example 2. Consider the equation

$$
\left(y^{\prime \prime}-y\right)\left(y^{\prime \prime}-y^{\prime}\right)=0
$$

Here we have $\lambda_{1}=y, \lambda_{2}=p$. Let

$$
a=p^{2}-x^{2}, \quad b=x-\ln (p+y), \quad f=y-p, \quad g=p e^{-x} .
$$

The pair of associated ODEs is

$$
b^{\prime \prime}=-\frac{b^{\prime}\left(2+a b^{\prime}\right)}{a} \quad \text { and } \quad b^{\prime \prime}=\frac{b^{\prime}}{a} .
$$

Both of them are point equivalent to the ODE $b^{\prime \prime}=0$.

Example 3. Consider the equation

$$
\left(y^{\prime \prime}+x\right)\left(y^{\prime \prime}-1\right)=0,
$$


for which $\lambda_{1}=-x, \lambda_{2}=1$. Let

$$
a=\frac{1}{2} x^{2}+p, \quad b=\frac{1}{3} x^{3}-p x+y, \quad f=p-x, \quad g=\frac{1}{2} x^{2}-p x+y .
$$

The pair of associated ODEs is

$$
b^{\prime \prime}=\frac{1}{b^{\prime}-1} \quad \text { and } \quad b^{\prime \prime}=\frac{-1-4 b^{2}+2 \sqrt{b^{\prime 2}+4 b^{\prime 4}}}{\left(1+8 b^{\prime 2}-4 \sqrt{b^{\prime 2}+4 b^{\prime 4}}\right)^{3 / 2}} .
$$

Both of them admit the same Lie algebra, hence they are point equivalent.

Example 4. We give the example of two pairs of functions in general position for which associated ODEs are not point equivalent to each other. Let functions $(a, b)$ and $(f, g)$ satisfy the conditions

$$
a^{2}-2 a b+f^{2}+2 b f-g^{2}+b^{2}=1, \quad g \geqslant 0, \quad b-a+f \geqslant 0 .
$$

Then $g=h(a, b, f)=\sqrt{a^{2}-2 a b+f^{2}+2 b f+b^{2}-1}$, and the associated equation is

$$
b^{\prime \prime}=\frac{b^{\prime}}{a-b}-\frac{2 b^{\prime 2}}{a-b}+\frac{b^{\prime 3}}{a-b} .
$$

It is point equivalent to the equation $b^{\prime \prime}=0$.

On the other hand, $b=h(f, g, a)=a-f+\sqrt{1-2 a f+g^{2}}$, and the second associated equation is not cubic in the first derivative, hence it is not point equivalent to the first one.

In conclusion we give the formulas needed to calculate the invariants used in Tresse-Kruglikov theorem. We denote $\frac{\partial}{\partial a}, \frac{\partial}{\partial b}$ and $\frac{\partial}{\partial c}$ by $\nabla_{a}, \nabla_{b}$ and $\nabla_{c}$. In the initial coordinates $(x, y, p)$ they are of the form

$$
\begin{aligned}
& \nabla_{a}=\frac{1}{\Delta}\left(\left(b_{y} f_{p}-b_{p} f_{y}\right) \frac{\partial}{\partial x}+\left(b_{p} f_{x}-b_{x} f_{p}\right) \frac{\partial}{\partial y}+\left(b_{x} f_{y}-f_{x} b_{y}\right) \frac{\partial}{\partial p}\right) \\
& \nabla_{b}=\frac{1}{\Delta}\left(\left(a_{p} f_{y}-a_{y} f_{p}\right) \frac{\partial}{\partial x}+\left(a_{x} f_{p}-a_{p} f_{x}\right) \frac{\partial}{\partial y}+\left(a_{y} f_{x}-a_{x} f_{y}\right) \frac{\partial}{\partial p}\right) \\
& \nabla_{c}=\frac{1}{\Delta}\left(\left(a_{y} b_{p}-a_{p} b_{y}\right) \frac{\partial}{\partial x}+\left(a_{p} b_{x}-a_{x} b_{p}\right) \frac{\partial}{\partial y}+\left(a_{x} b_{y}-a_{y} b_{x}\right) \frac{\partial}{\partial p}\right)
\end{aligned}
$$

where

$$
\Delta=a_{p} b_{x} f_{y}-a_{p} b_{y} f_{x}-a_{y} p_{x} f_{p}+a_{y} b_{p} f_{x}+a_{x} b_{y} f_{p}-a_{x} b_{p} f_{y}
$$

and the partial derivatives of $f$ in $x, y$ and $p$ are understood as derivatives of a composite function.

The invariant $H$ is

$$
\begin{array}{r}
\widetilde{H}=\nabla_{c}^{2} \nabla_{a}^{2} F-2 \cdot \frac{h_{a}}{h_{b}} \cdot \nabla_{c}^{2} \nabla_{a} \nabla_{b} F+\left(\frac{h_{a}}{h_{b}}\right)^{2} \cdot \nabla_{c}^{2} \nabla_{b}^{2} F-4 \nabla_{c} \nabla_{a} \nabla_{b} F+4 \cdot \frac{h_{a}}{h_{b}} \cdot \nabla_{c} \nabla_{b}^{2} F+ \\
+6 \nabla_{b}^{2} F+F\left(2 \nabla_{c}^{3} \nabla_{a} F-2 \cdot \frac{h_{a}}{h_{b}} \cdot \nabla_{c}^{3} \nabla_{b} F-3 \nabla_{c}^{2} \nabla_{b} F\right)-\nabla_{c} F\left(\nabla_{c}^{2} \nabla_{a} F-\frac{h_{a}}{h_{b}} \cdot \nabla_{c}^{2} \nabla_{b} F-\right. \\
\left.\quad-4 \nabla_{c} \nabla_{b} F\right)+\nabla_{c}^{3} F\left(\nabla_{a} F-\frac{h_{a}}{h_{b}} \cdot \nabla_{b} F\right)-3 \nabla_{c}^{2} F \cdot \nabla_{b} F+F^{2} \cdot \nabla_{c}^{4} F .
\end{array}
$$


Invariant differential operators are

$$
\begin{aligned}
\Delta_{c}= & \widetilde{\mathcal{D}}_{c}+(r-s) \frac{\nabla_{c}^{5} F}{5 \nabla_{c}^{4} F}, \\
\Delta_{a}= & \widetilde{\mathcal{D}}_{a}-\frac{h_{a}}{h_{b}} \cdot \widetilde{\mathcal{D}}_{b}+F \widetilde{\mathcal{D}}_{c}+(3 r+2 s) \nabla_{c} F+ \\
& +(2 r+s)\left(\frac{F \cdot \nabla_{c}^{5} F+\nabla_{c}^{4} \nabla_{a} F-\frac{h_{a}}{h_{b}} \cdot \nabla_{c}^{4} \nabla_{b} F}{\nabla_{c}^{4} F}\right), \\
\Delta_{b}= & \frac{\nabla_{c}^{5} F}{5 \nabla_{c}^{4} F} \cdot \widetilde{\mathcal{D}}_{a}+\left(1-\frac{h_{a}}{h_{b}} \cdot \frac{\nabla_{c}^{5} F}{5 \nabla_{c}^{4} F}\right) \widetilde{\mathcal{D}}_{b}+ \\
& +\left(2 \nabla_{c} F+\frac{6 F \cdot \nabla_{c}^{5} F+5 \nabla_{c}^{4} \nabla_{a} F-5 \cdot \frac{h_{a}}{h_{b}} \cdot \nabla_{c}^{4} \nabla_{b} F}{\nabla_{c}^{4} F}\right)+(3 r+2 s) \frac{\nabla_{c}^{2} F}{8}+ \\
& +(r+2 s) \frac{\nabla_{c}^{4} \nabla_{b} F}{4 \nabla_{c}^{4} F}+(19 r+6 s) \frac{\nabla_{c}^{5} F \cdot \nabla_{c} F}{10 \nabla_{c}^{4} F}+(21 r+6 s) \frac{\nabla_{c}^{5} F\left(F \cdot \nabla_{c}^{5} F+\nabla_{c}^{4} \nabla_{b} F\right)}{20 \nabla_{c}^{4} F \cdot \nabla_{c}^{4} F},
\end{aligned}
$$

where $\widetilde{\mathcal{D}}_{a}, \widetilde{\mathcal{D}}_{b}, \widetilde{\mathcal{D}}_{c}$ - are the total derivatives corresponding to the operators $\nabla_{a}, \nabla_{b}, \nabla_{c}$.

Acknowledgement. Author wishes to express his deep gratitude to V.V. Lychagin for the formulation of the problem and valuable comments.

\section{References}

[1] V.I. Arnold, Geometrical Methods in the Theory of Ordinary Differential Equations. Springer Berlin Heidelberg, 2008, 368 p.

[2] N. Ibragimov, A practical course in differential equations and mathematical modelling. Classical and new methods, nonlinear mathematical models, symmetry and invariance principles, Higher Education Press, Beijing (P.R. China) and World Scientific (Singapore), 2009, 348 p.

[3] N. Ibragimov, F. Magri, Geometric proof of Lie's linearization theorem, Nonlinear Dynamics, Vol. 36 (2004), 41-46.

[4] B. Kruglikov, Point classification of 2nd order ODEs: Tresse classification revisited and beyond. Differential equations: Geometry, Symmetries and Integrability: The Abel Symposium 2008, Abel Symposia 5, Berlin, Springer, 2009, 199-221.

[5] S. Lie, Klassifikation und Integration von gewöhnlichen Differentialgleichungen zwischen $x$, y, die eine Gruppe von Transformationen gestatten, III, Archiv for Mathematik og Naturvidenskab 8 (Kristiania, 1883), 371-458; Gesam. Abh. Bd., Vol. 5 (1924), paper XIV, 362-427.

[6] R. Liouville, Sur les invariants de certaines équations différentielles et sur leurs applications, Journal de l'École Polytechnique, Vol. 59 (1889), 7-76.

[7] O. I. Morozov, Point equivalence problem for the second order ordinary differential equations, Nauch. vestn. MGTU GA, Vol. 157 (2006), 90-104.

[8] A. Tresse, Sur les invariants différentiels des groupes continus de transformations, Acta Math., Vol. 18 (1894), 1-88. 
[9] A. Tresse, Détermination des invariants ponctuels de l'équation différentielle ordinaire du second ordre $y^{\prime \prime}=\omega\left(x, y, y^{\prime}\right)$, Leipzig (1896).

[10] V. Yumaguzhin, On the obstruction to linearizability of 2-order ordinary differential equations, Acta Applicandae Mathematicae, Vol. 83, No. 1-2 (2004), 133-148.

[11] V. Yumaguzhin, Differential invariants of 2-order ODEs, I, Acta Applicandae Mathematicae, Vol. 109 (2010), 283-313. 